\title{
A New Look at the GEO and Near-GEO Regimes: Operations, Disposals, and Debris
}

\author{
Nicholas L. Johnson \\ NASA Johnson Space Center
}

Since 1963 more than 900 spacecraft and more than 200 launch vehicle upper stages have been inserted into the vicinity of the geosynchronous regime. Equally important, more than 300 spacecraft have been maneuvered into disposal orbits at mission termination to alleviate unnecessary congestion in the finite GEO region. However, the number of GEO satellites continues to grow, and evidence exists of a substantial small debris population. In addition, the operational modes of an increasing number of GEO spacecraft differ from those of their predecessors of several decades ago, including more frequent utilization of inclined and eccentric geosynchronous orbits. Consequently, the nature of the GEO regime and its immediate surroundings is evolving from well-known classical characteristics. This paper takes a fresh look at the GEO satellite population and the near- and far-term environmental implications of the region, including the effects of national and international debris mitigation measures. 


\title{
IAC-11.A6.2.7
}

\section{A NEW LOOK AT THE GEO AND NEAR-GEO REGIMES: OPERATIONS, DISPOSALS, AND DEBRIS}

\author{
Nicholas L. Johnson \\ Chief Scientist for Orbital Debris, NASA, Houston, Texas, USA \\ Nicholas.L.Johnson@nasa.gov
}

Since 1963 more than 900 spacecraft and more than 200 launch vehicle upper stages have been inserted into the vicinity of the geosynchronous regime. Equally important, more than 300 spacecraft have been maneuvered into disposal orbits at mission termination to alleviate unnecessary congestion in the finite GEO region. However, the number of GEO satellites continues to grow, and evidence exists of a substantial small debris population. In addition, the operational modes of an increasing number of GEO spacecraft differ from those of their predecessors of several decades ago, including more frequent utilization of inclined and eccentric geosynchronous orbits. Consequently, the nature of the GEO regime and its immediate surroundings is evolving from well-known classical characteristics. This paper takes a fresh look at the GEO satellite population and the near- and far-term environmental implications of the region, including the effects of national and international debris mitigation measures.

\section{EVOLUTION OF THE GEOSYNCHRONOUS ENVIRONMENT}

The geosynchronous orbital regime represents a unique vantage point from which to conduct a wide variety of applications missions, e.g., communications, navigation, and national defense, in addition to scientific investigations. At a mean altitude of $35,786 \mathrm{~km}$, a satellite will circle the Earth every 1436 minutes and will remain in lock-step with the rotation of the Earth as it revolves about the Sun. A circular or nearly circular geosynchronous orbit (GEO) with an orbital inclination of zero degrees is known as a geostationary orbit.

The Inter-Agency Space Debris Coordination Committee (IADC) has defined a GEO protected region as that volume of space bounded in altitude by $200 \mathrm{~km}$ below the GEO altitude to $200 \mathrm{~km}$ above the GEO altitude and in latitude by 15 degrees on either side of the equator. ${ }^{1}$ This region was originally conceived to include a nominal operational altitude excursion of GEO +/- $75 \mathrm{~km}$ and an additional $125 \mathrm{~km}$ on either side for a satellite translational corridor.

The SYNCOM 2 spacecraft was the first operational man-made satellite to be inserted into an inclined, nearly circular geosynchronous orbit in 1963 and was followed by the 1964 deployment of the SYNCOM 3 spacecraft into a true geostationary orbit. These very modest 39-kg satellites have now been replaced by vastly more capable vehicles with 100 times the initial on-station mass (Figure 1). The launch of more than 900 spacecraft into geosynchronous orbits during the past half century (Figure 2) has been accompanied by more than 230 launch vehicle stages or apogee kick motors and a relatively small number of mission-related debris, which have taken up residence within or near the GEO regime.

Of course, some of the above objects were never inserted into a true geosynchronous orbit, and others have been transferred from the GEO region to disposal orbits. As of January 2011, the number of cataloged objects which remained completely within $200 \mathrm{~km}$ of the GEO altitude was about 650 . Of these only a little more than half were operational spacecraft; the rest were non-functional spacecraft, derelict propulsion stages, or smaller debris. In addition, nearly another 700 cataloged objects were in elliptical orbits which passed through the GEO +/- $200 \mathrm{~km}$ altitude.

Despite the increasing size and capability of GEO spacecraft, the number of operational satellites in this region has steadily grown. Until 1999 the average annual increase in the number of operational GEO 
spacecraft was 7-8, but during the next dozen years the rate rose to 12 per annum (Figure 3). Today, more than 400 GEO spacecraft are operational. The nature of these orbits is the subject of the next section.

\section{INCLINED AND ECCENTRIC GEOSYNCHRONOUS ORBITS}

With some exceptions, during the early years of exploitation of the GEO regime, operational GEO spacecraft tended to be very close to geostationary with small inclinations and eccentricities. This condition was a function as much of terrestrial operational constraints as of spacecraft limitations. However, the station-keeping requirements to off-set solar-lunar gravitational perturbations to maintain a near-zero orbital inclination (North-South station-keeping) are much greater than those to maintain an assigned longitude (East-West station-keeping). Consequently, to conserve propellants a significant number of GEO spacecraft are now operating in inclined orbits.

The first spacecraft in the U.S. Tracking and Data Relay Satellite (TDRS) system was launched in 1983. By the start of 1991 the average inclination of the constellation was only 1.9 degrees, but grew to 5.0 degrees and 7.9 degrees 10 and 20 years later, respectively. Figure 4 is an illustration of the TDRS daily tracks today with five of the eight spacecraft possessing inclinations in excess of 10 degrees.

The former Soviet Union pioneered a technique of employing solar-lunar gravitational perturbations to the advantage of GEO spacecraft operators. ${ }^{2}$ By carefully selecting the right ascension of the initial orbital plane along with an inclination of a few degrees, such perturbations will naturally reduce the inclination to zero before increasing again. Figure 5 indicates the evolution of the inclination of the Raduga 17 spacecraft during its operational lifetime. In fact, all Soviet GEO spacecraft launched from 1974 through 1993 (more than 100 in all), performed no North-South station-keeping at all. As the mean lifetime of Soviet spacecraft increased, so, too, did the initial orbital inclination to constrain the value of the inclination near end-of- mission.

As of January 2011, 25\% of all operational GEO satellites had inclinations greater than 1 degree (Figures 3 and 6). For GEO spacecraft reaching end-of-mission, the statistics are even more dramatic. During the period 2006 through 2009, when more than 60 GEO spacecraft were retired, fully two-thirds had inclinations greater than 1 degree.

With relatively rare exceptions, the eccentricities of GEO spacecraft are normally maintained at very low values, regardless of the age of the spacecraft. More than 95\% of the operational GEO spacecraft in January 2011 exhibited differences in their apogee and perigee values of less than $100 \mathrm{~km}$. This equates to an eccentricity of less than 0.001 and is well within the IADC $150 \mathrm{~km}$-tall operational corridor for GEO spacecraft.

However, beginning in the year 2000, new classes of GEO networks, exhibiting high inclinations and low to moderate eccentricities, have been deployed (Figure 7). The first of these was the 3-member U.S. Sirius communications constellation with inclinations between 60 and 65 degrees and eccentricities of 0.27. In 2010 the first Chinese Beidou navigation GEO satellite in an inclined orbit appeared with an inclination of 55 degrees and eccentricity of 0.003 . Four of these spacecraft had been launched through July, 2011. Finally, the first Japanese Michibiki quasi-zenith satellite debuted in 2010 with an inclination of 41 degrees and an eccentricity of 0.075 .

One of the consequences of the overall increasing use of higher inclinations for GEO spacecraft, as well as the new high eccentricity satellite networks, is an increase in the relative velocity among objects in the GEO region. This leads to higher collision probabilities and to more severe damage when collisions do occur. $^{3}$

\section{THE DISPOSAL OF GEO SPACECRAFT}

In 1977, when the accumulated number of spacecraft and launch vehicle stages in or near the GEO region exceeded 100, Perek called upon the aerospace community to implement a policy to maneuver GEO 
spacecraft at end-of-mission to disposal orbits where they could not interfere with operational satellites. ${ }^{4}$ Some satellite operators, e.g., INTELSAT, heeded this call, and by 1993 the International

Telecommunication Union (ITU) had issued its first formal recommendation in the matter. ${ }^{5}$ A general agreement was reached that the disposal orbits should be situated above the GEO region, rather than below it, to avoid interference with objects in geosynchronous transfer orbits. The recommended minimum height of the disposal orbit grew, from $50 \mathrm{~km}$ to $150 \mathrm{~km}$ to $300 \mathrm{~km}$ or more, as an appreciation for the complexity of perturbations on GEO disposal orbits evolved. ${ }^{6}$

After many years of deliberations, the IADC concluded that a simpler, results-oriented approach was best, and thus the organization revised its GEO spacecraft disposal recommendation in 2007. The essence of the new recommendation is that spacecraft should be maneuvered to disposal orbits sufficiently high that the satellites will remain above the GEO protected region, i.e., above the GEO altitude $+200 \mathrm{~km}$, despite the influence of solar-lunar gravitational perturbations and solar radiation pressure. NASA has accepted this recommendation and assesses compliance if the spacecraft is not projected to come within the GEO protected zone for at least 100 years. $^{7}$

During the period 2001-2010, more than 160 GEO spacecraft reached their end-of-mission, and of these approximately $80 \%$ moved into disposal orbits, indicating a strong degree of support by the satellite operator community for the preservation of the GEO region. Some of the spacecraft which were left in the GEO region experienced a sudden catastrophic malfunction which prevented the execution of a disposal maneuver, e.g., ECHOSTAR 2, GALAXY 3R, INTELSATs 511 and 804, TELSTAR 4, and UFO 3.

Despite the high percentage of disposal maneuvers conducted, not all met the altitude recommendations of the IADC or the ITU. One mitigating factor is that many of the retiring spacecraft were designed and built (and some even launched) before the current disposal recommendations were established. Hence, application of these recommendations to older systems was not always practical or possible. On the other hand, several very old spacecraft were still able to comply with the present recommendations. For example, the 27-year-old TDRS 1 and the 32-year-old MARISAT 2 spacecraft reached disposal orbits of $345 \mathrm{~km}$ by $525 \mathrm{~km}$ and $330 \mathrm{~km}$ by $1205 \mathrm{~km}$ above the GEO altitude, respectively.

Figure 8 depicts the initial minimum altitude above GEO for all spacecraft retiring during 20012010. Overall, $70 \%$ of the maneuvering spacecraft were transferred to orbits at least $200 \mathrm{~km}$ above the GEO altitude, and nearly $50 \%$ reached at least $300 \mathrm{~km}$ above the GEO altitude. The magnitude of future altitude variations, e.g., excursions below GEO $+200 \mathrm{~km}$, is dependent upon the mass, size, shape, and stability of the spacecraft, as well as its initial orbital plane with respect to the Sun and the Moon.

One of the often expressed concerns with the concept of disposal orbits for GEO spacecraft involves the creation of a new "ring" of spacecraft about $300 \mathrm{~km}$ above the GEO altitude. Such a concentration of derelict objects might give rise to future collisions which would rain debris down through the GEO region. If all retiring GEO spacecraft were indeed placed into nearly circular orbits near GEO + $300 \mathrm{~km}$, then the probability of future collisions would continue to increase. However, in practice a wide variety of disposal orbits are employed, in part since GEO spacecraft have varying amounts of residual propellants when their missions are terminated. Figure 9 shows the distinct orbital variation of cataloged objects now with perigees above GEO + $200 \mathrm{~km}$.

In addition to curtailing the accumulation of derelict spacecraft in the GEO region, consideration must also be given to limiting the presence of launch vehicle upper stages, spacecraft apogee kick motors, and other mission-related debris. During the decade of the 1980's, nearly 90 upper stages or apogee kick motors were deposited in or near the GEO region, for an average rate of 9 per year. By contrast, during 2010 only two upper stages accompanied 32 spacecraft to the vicinity of the GEO region. This significant improvement has primarily been possible with the increased use of single, integrated spacecraft propulsion systems which perform both geosynchronous orbit insertion maneuvers and orbital maintenance maneuvers. Most spacecraft are also designed now to eliminate the release of sensor covers and other debris. 


\section{SMALLER DEBRIS IN AND NEAR THE GEO REGION}

To a great extent, our understanding of the GEO and near-GEO satellite populations is centered on the large spacecraft and separate propulsion units there. The primary source of this information is the U.S. Space Surveillance Network (SSN), which employs quite capable radars and electro-optical sensors. However, in general, objects smaller than $0.8 \mathrm{~m}$ in diameter have not been cataloged. Only a total of 19 small debris currently appear in the official U.S. Satellite Catalog: 12 represent presumed fragmentation debris, while the other 7 objects are known or appear to be mission-related debris. A recent report from the European Space Agency indicates that as many as 3 apogee kick motors and 63 mission-related debris have not been cataloged by the SSN. ${ }^{8}$

Recently, another source of information on debris in and near the GEO region has been the International Scientific Optical Network (ISON), managed by the Keldysh Institute of Applied Mathematics, which is part of the Russian Academy of Sciences. The ISON database of 1 February 2011was tracking a total of 1557 objects, including 378 small debris which were not spacecraft or propulsion units. ${ }^{9}$

For over a decade, NASA has sponsored observations of the near-GEO regime for the express purpose of detecting small debris, i.e., debris between 25 and $100 \mathrm{~cm}$. Under a grant from NASA, the University of Michigan's 61-cm aperture Curtis-Schmidt telescope at Cerro Tololo Inter-American Observatory in Chile is used to discover and to characterize objects near GEO which are not in the U.S. Satellite Catalog. When engaged in these activities, the telescope is referred to as the Michigan Orbital DEbris Survey Telescope (MODEST).

Figure 10 provides an assessment of the population of objects in and near the GEO region, derived from several years of data from MODEST. The curve labeled UCT depicts uncataloged objects only, whereas the second curve (CT +UCT) combines the uncataloged with the cataloged objects for a complete population. The UCT curve drops off after $17^{\text {th }}$ absolute magnitude (approximately $25 \mathrm{~cm}$ ) due to sensor limitations; the population likely continues to increase as the size becomes smaller. The diagonal line represents the slope of debris sizes one would expect from an explosion and was derived from observations of exploded spacecraft and rocket bodies in low Earth orbit. The fact that the slope of the diagonal line is very similar to the slope of the UCT curve is suggestive that these small objects originated from explosions in the GEO region. To date, only two satellite explosions (one a spacecraft and one a rocket body) have been confirmed in the GEO region, although other explosions might also have occurred.

A cumulative population distribution for the GEO region is shown in Figure 11. The number of objects between 10 and $100 \mathrm{~cm}$ is thought to be at least twice the number of the 1 meter and larger population. In early 2012 NASA's Meter-Class Autonomous Telescope (MCAT) is expected to begin operational surveys of the GEO region with an ability to detect debris as small as $10 \mathrm{~cm}$.

An interesting aspect of numerous small debris in or near the GEO region is their large area-to-mass ratio, i.e., larger than $1 \mathrm{~m}^{2} / \mathrm{kg}$. Such objects are highly susceptible to the effects of solar radiation pressure as exhibited by dramatic changes in inclination and eccentricity. In turn, these orbital parameter perturbations can affect both the probability of collision and the consequence (i.e., impact velocity) of collisions within GEO.

\section{SUMMARY}

The number of both operational spacecraft and debris in and near the GEO region continues to increase. Moreover, the orbital characteristics of these objects can vary dramatically from the classical geostationary case, in part due to the intentional use of significantly inclined and eccentric orbits and in part due to natural orbital perturbations. Recent dedicated surveys of the GEO region have revealed a substantial number of small, but still potentially hazardous, debris which are not in official satellite catalogs. The responsible disposal of GEO spacecraft at end-of-mission is now commonplace and serves to preserve the GEO region for future operations. 


\section{REFERENCES}

1. IADC Space Debris Mitigation Guidelines, Inter-Agency Space Debris Coordination Committee, IADC02-01, 2002; revised 2007.

2. Johnson, N.L., "The Development and deployment of Soviet geosynchronous satellites", Journal of the British Interplanetary Society, 35, 1982, pp. 450-458.

3. McKnight, D.S. and Di Pentio, F., "New Insights on the Orbital Debris Collision Hazard at GEO”, IAC11.A6.2.6, 62 ${ }^{\text {nd }}$ International Astronautical Congress, Cape Town, South Africa, 3-7 October 2011.

4. Perek, L., "Physics, uses and regulation of GSO”, IAF-SL-77-44, $28^{\text {th }}$ Congress of the International Astronautical Federation, Prague, 1977.

5. Johnson, N.L., "Protecting the GEO environment: policies and practices”, Space Policy, 15, 1999, pp. 127-135.

6. Support Document to the IADC Space Debris Mitigation Guidelines, Inter-Agency Space Debris Coordination Committee, IADC-04-06, 2004.

7. Process for Limiting Orbital Debris, NASA Standard 8719.14, NASA, 2007.

8. Flohrer, T., Choc, R., and Bastida, B., Classification of Geosynchronous Objects, Issue 13, European Space Agency, 2011.

9. Agapov, V., "Review of events occurred in geostationary region in 2010 based on data obtained by ISON international network", presentation to the $48^{\text {th }}$ session of the United Nations Committee on the Peaceful Uses of Outer Space, Scientific and Technical Subcommittee, Vienna, 2011. 


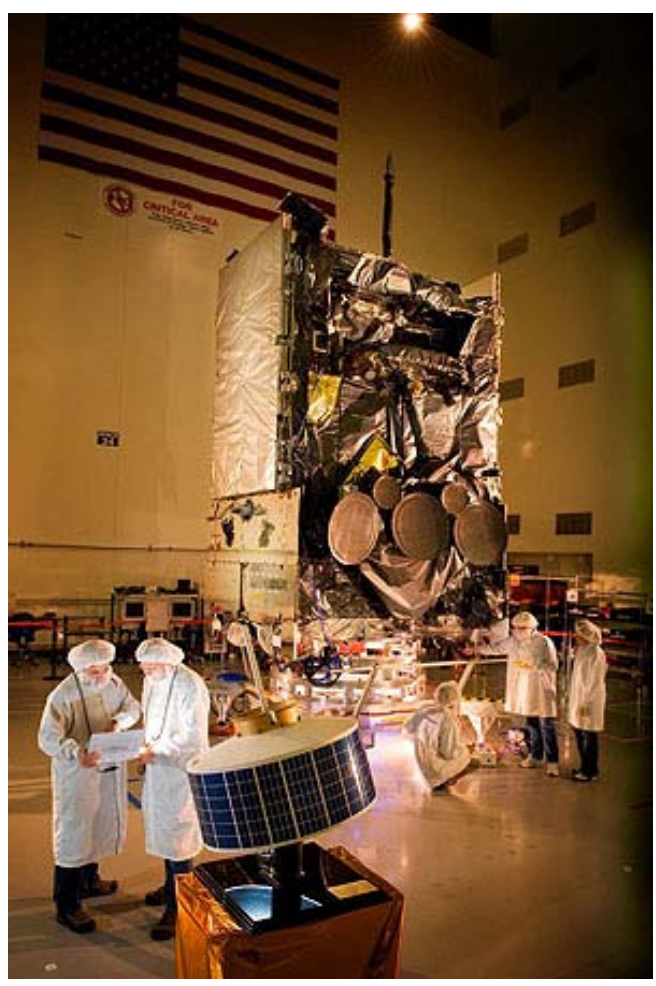

Fig. 1. The first generation geosynchronous satellite, SYNCOM, is dwarfed by the new Boeing 702 series spacecraft (Credit: Boeing).

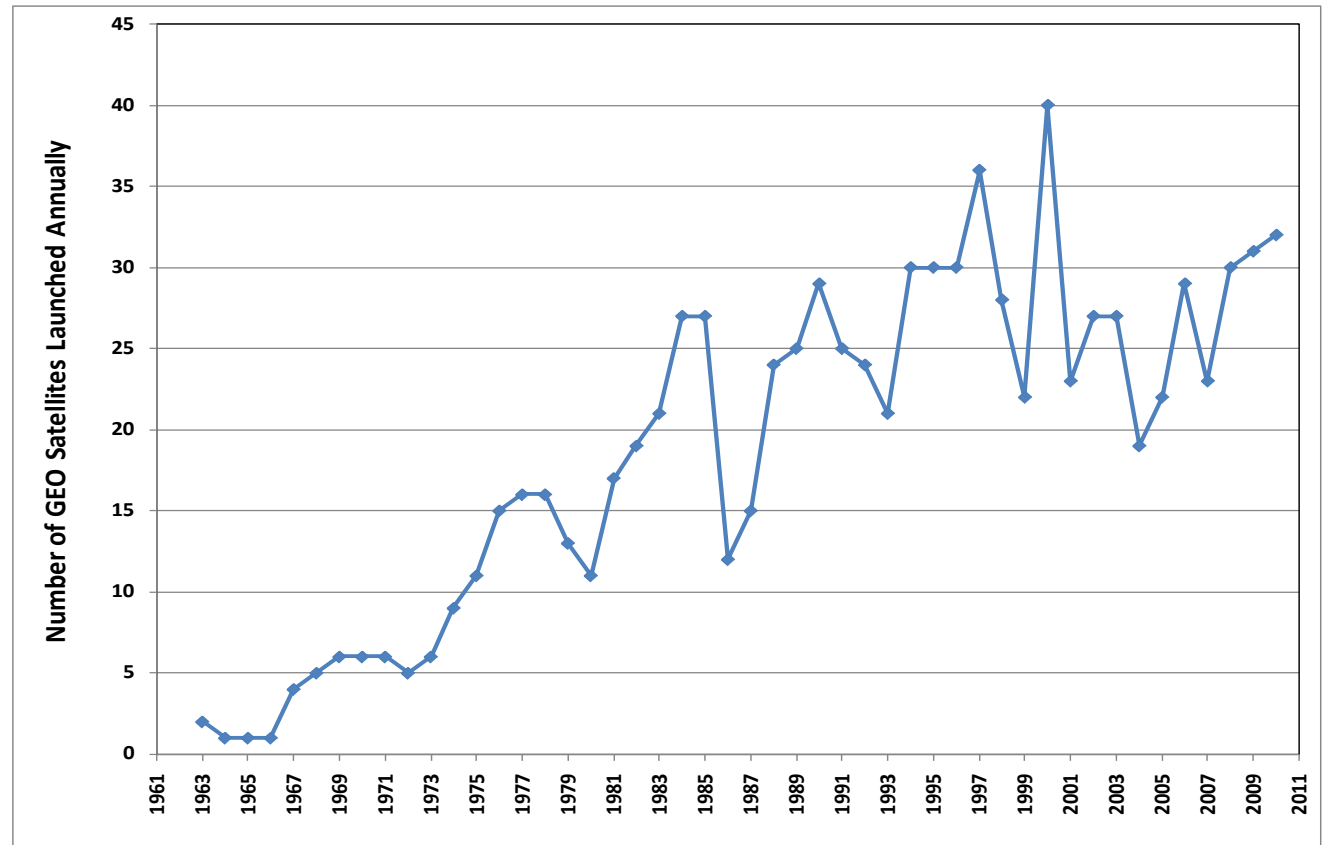

Fig. 2. During the past decade 26 spacecraft on average have been placed into geosynchronous orbits annually. 

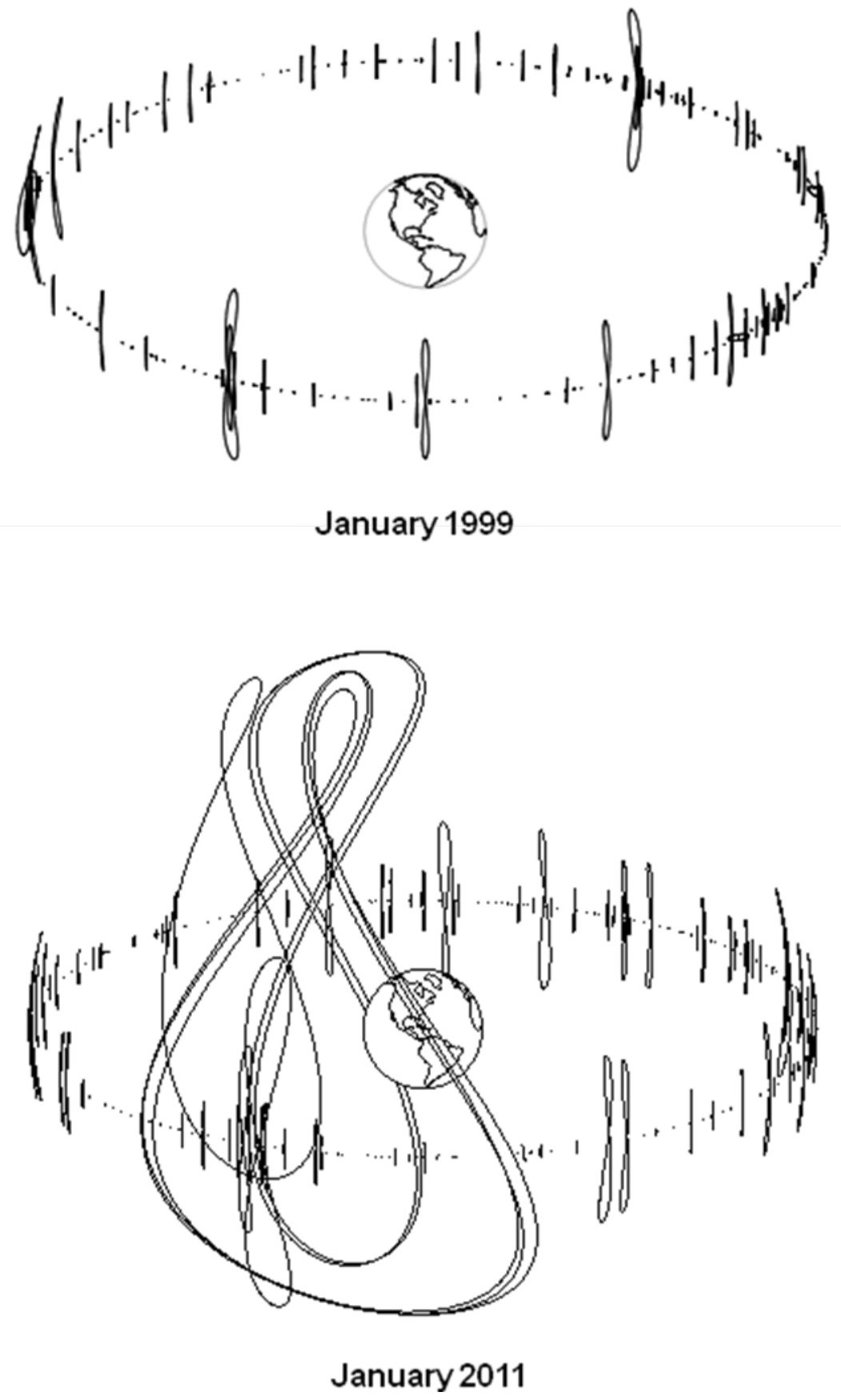

Fig. 3. The number and complexity of geosynchronous orbits for operational spacecraft increased significantly from 1999 to 2011. Only spacecraft whose orbital parameters are available at www.spacetrack.org are shown above. 


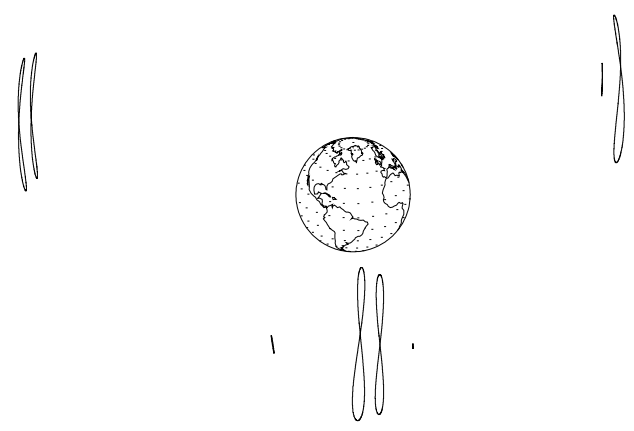

Fig 4. The five of the eight members of the TDRS network had inclinations greater than 10 degrees in January 2011.

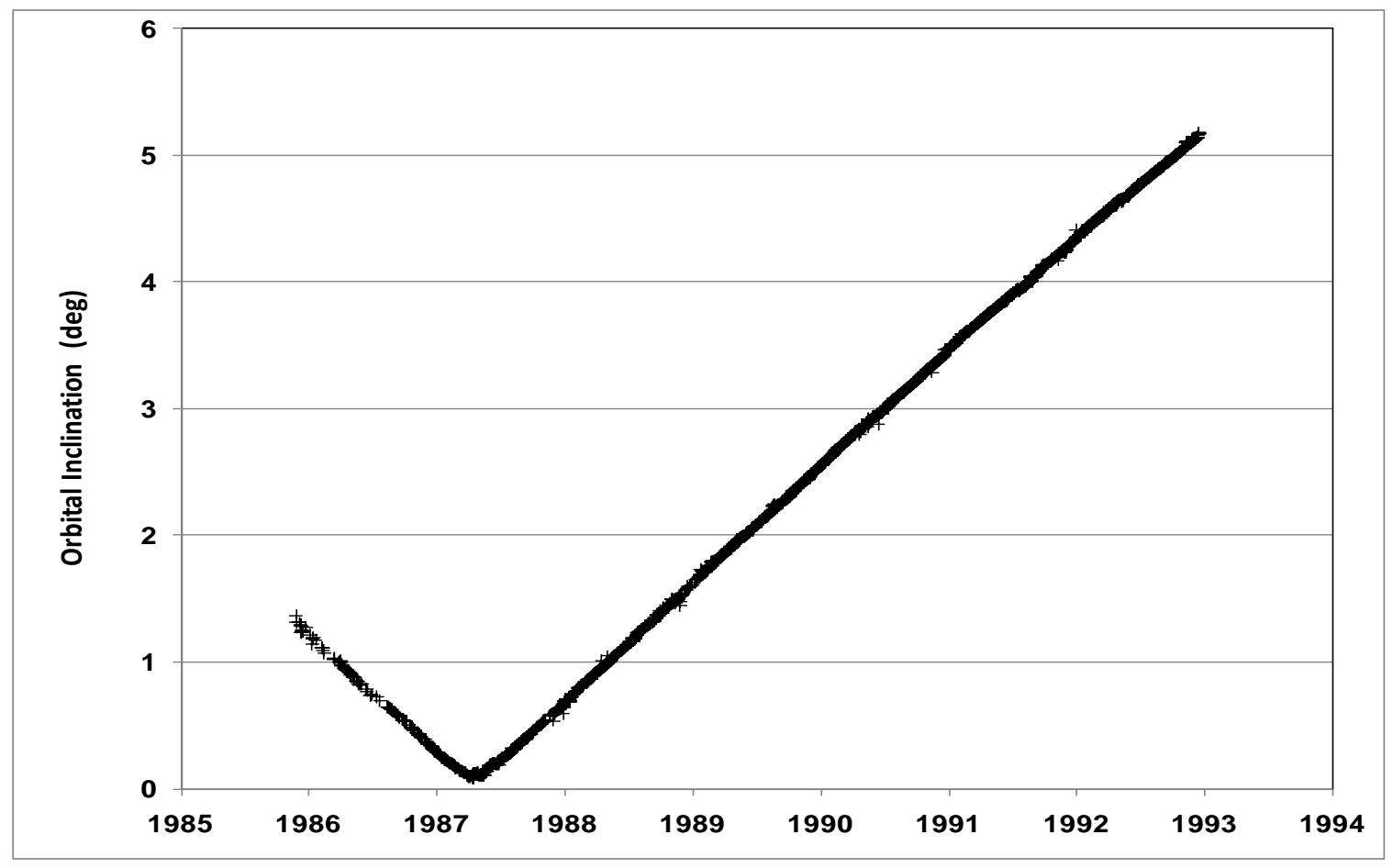

Fig 5. The natural perturbation of the inclination of the Raduga 17 spacecraft during its operational lifetime. 


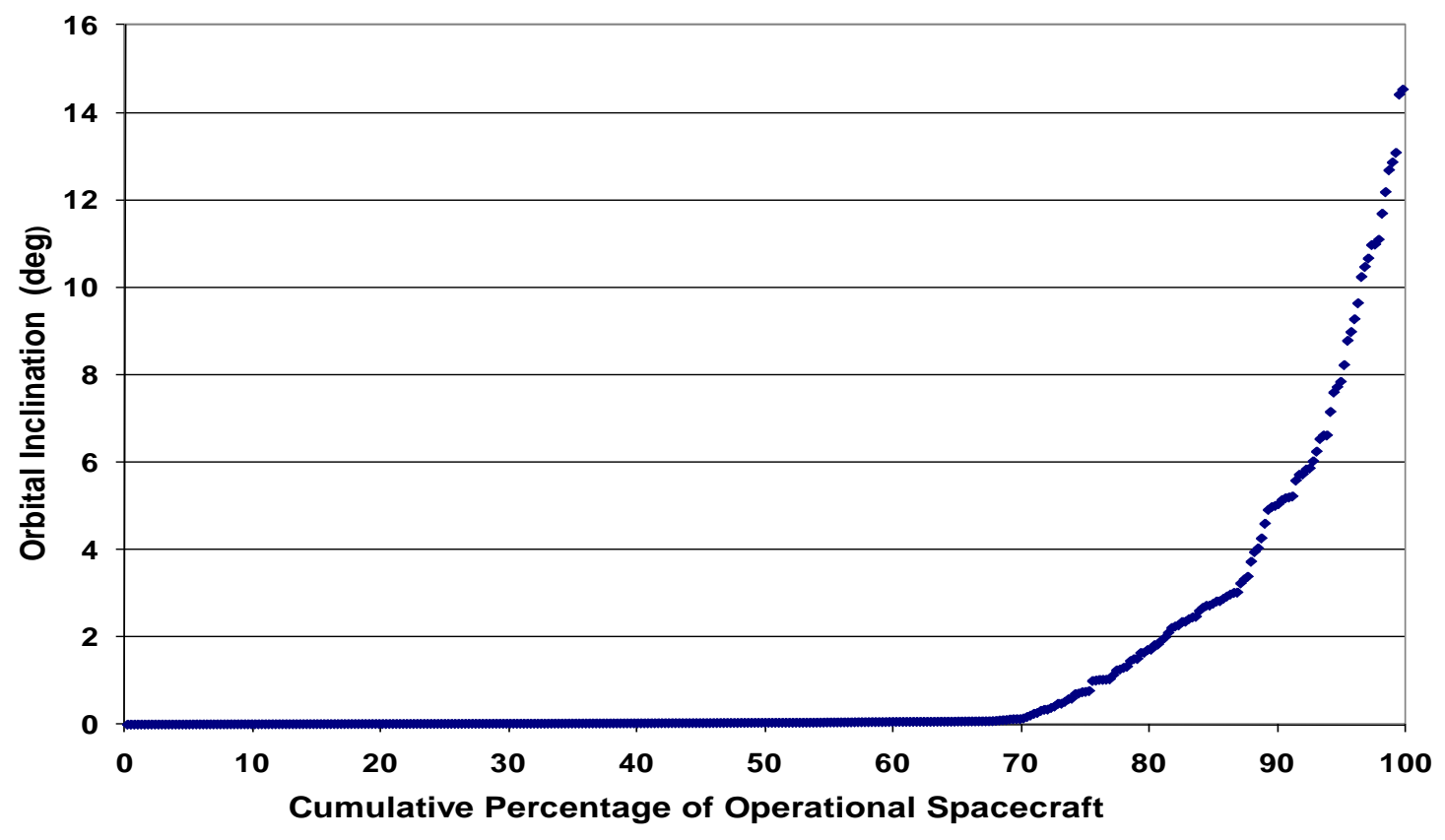

Fig. 6. One-fourth of all operational GEO satellites had orbital inclinations in excess of 1 degree in January 2011.

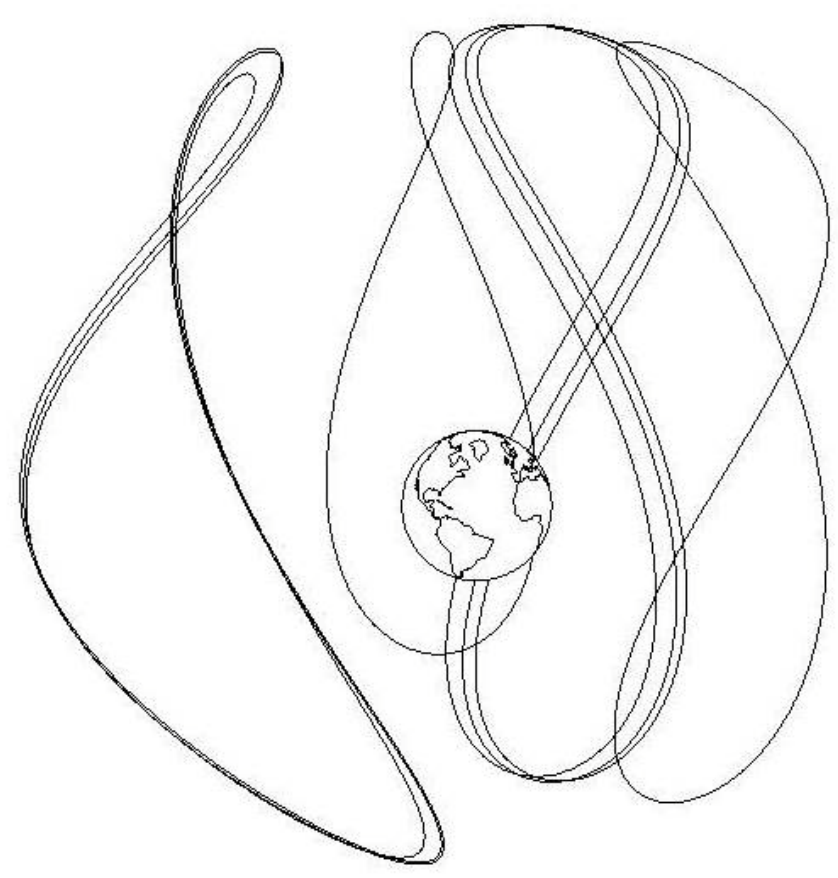

Fig. 7. Highly-inclined geosynchronous communications and navigations systems (Sirius, Beidou, and Michibiki) have been deployed since 2000. 


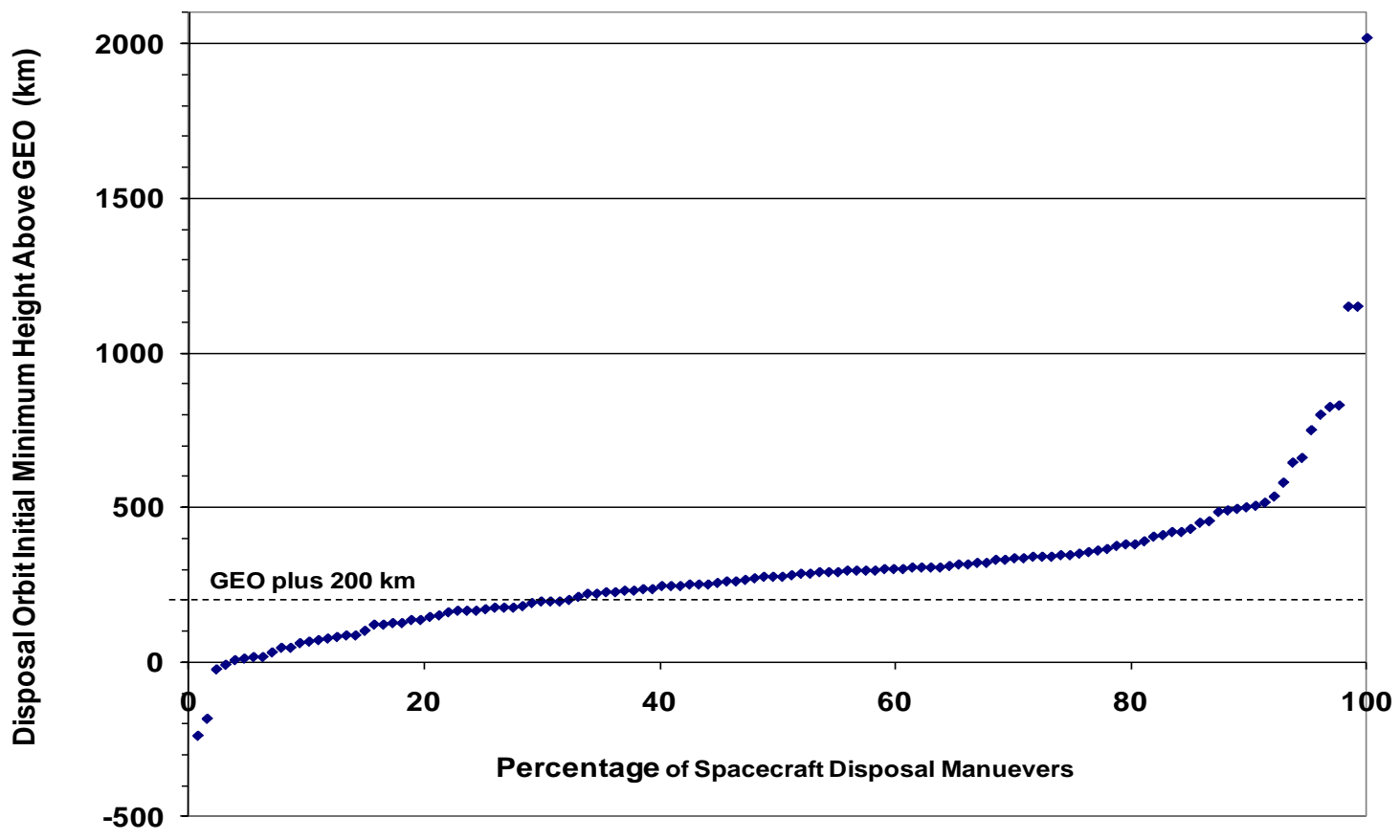

Fig. 8. Approximately 70\% of all GEO spacecraft disposal maneuvers during 2001-2010 resulted in a minimum altitude of at least $200 \mathrm{~km}$ above the GEO altitude.

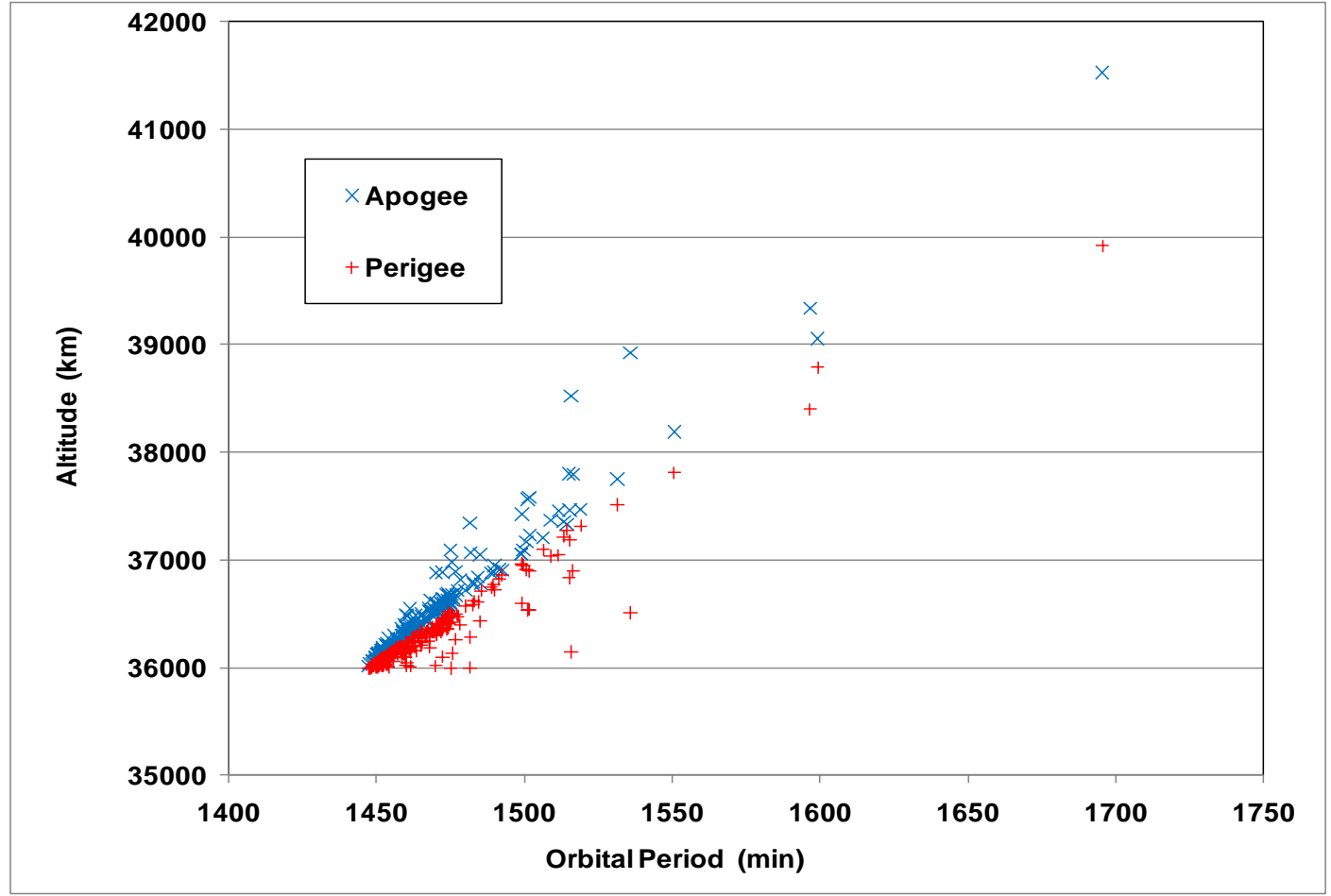

Fig 9. The distribution of all cataloged objects with perigees more than $200 \mathrm{~km}$ above the GEO altitude indicates a wide variety of orbits, not a concentration of disposal orbits. 


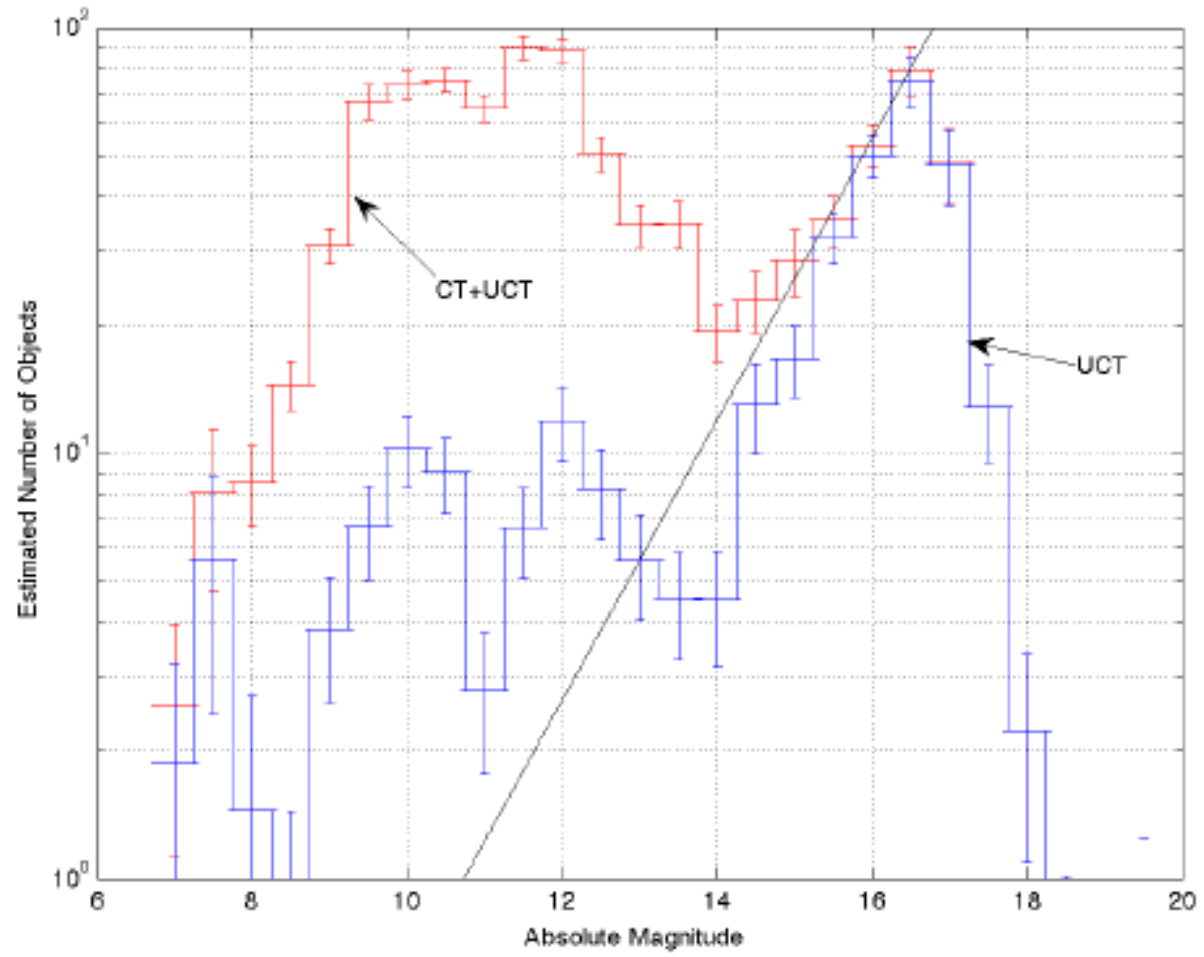

Fig. 10. Results from MODEST indicate a large number of small, uncataloged (UCT) objects near the GEO region. An object with $17^{\text {th }}$ absolute magnitude is assessed to be approximately $25 \mathrm{~cm}$ in size.

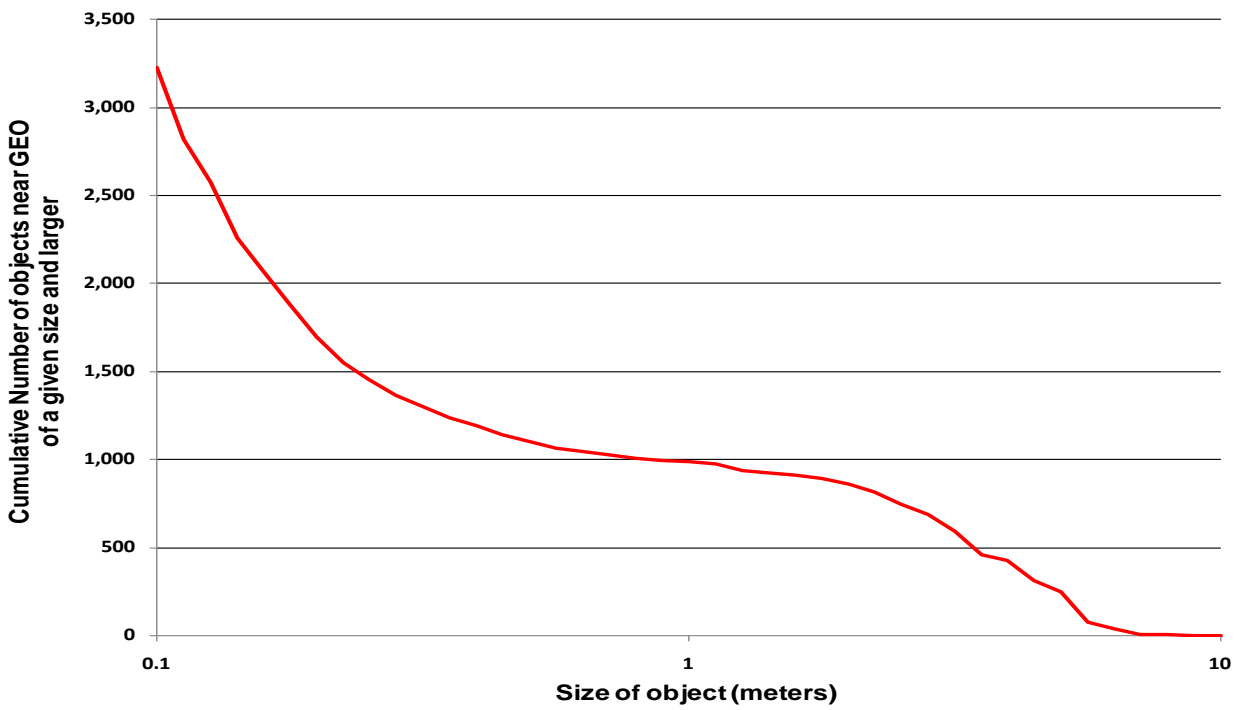

Fig. 11. Current NASA assessments of the smaller debris environment near the GEO region indicate a population about twice that of intact spacecraft and rocket bodies combined. 